\title{
AGAMOS: A Graph-Based Approach to Modulo Scheduling for Clustered Microarchitectures
}

\author{
Alex Aletà, Josep M. Codina, Jesús Sánchez, Member, IEEE, Antonio González, and David Kaeli
}

\begin{abstract}
This paper presents AGAMOS, a technique to modulo schedule loops on clustered microarchitectures. The proposed scheme uses a multilevel graph partitioning strategy to distribute the workload among clusters and reduces the number of intercluster communications at the same time. Partitioning is guided by approximate schedules (i.e., pseudoschedules), which take into account all of the constraints that influence the final schedule. To further reduce the number of intercluster communications, heuristics for instruction replication are included. The proposed scheme is evaluated using the SPECfp95 programs. The described scheme outperforms a state-of-the-art scheduler for all programs and different cluster configurations. For some configurations, the speedup obtained when using this new scheme is greater than 40 percent, and for selected programs, performance can be more than doubled.
\end{abstract}

Index Terms-Clustered microarchitectures, ILP, instruction replication, modulo scheduling, statically scheduled processors.

\section{INTRODUCTION}

LUSTERING is becoming a common trend in the design of current microprocessors due to its ability to reduce wire delays, power dissipation, and complexity. Clustering consists of partitioning processor resources into several groups or clusters. The components of each cluster are simpler, faster, and consume less power than a traditional centralized implementation. The resources in a cluster can be laid out in close proximity, which will reduce signal transmission delays [22]. Long (and slow) wires are used to interconnect clusters.

The use of clustering has been popular especially in the DSP market, including Texas Instruments' TMS320C6x [36], Analog Devices' TigerSHARC [19], BOPS's Man Array [31], HP/ST's Lx [17], and Equator's MAP1000 [20]. All of these processors use a statically scheduled, clustered microarchitecture.

The effectiveness of a statically scheduled processor depends heavily on the effectiveness of the compiler. Among the different compiler steps, code scheduling is probably the most critical for performance for this class of target processors. In this paper, we limit our focus to instruction scheduling techniques for clustered microprocessors. In particular, we focus on scheduling software-pipelined loops [12] since a vast majority of the execution time on this class of processors is spent in loop bodies.

- A. Aletà is with the Universitat Politècnica de Catalunya (UPC), Campus Nord, C6-221 Jordi Girona, 1, Barcelona, Spain 08034.

E-mail: aaleta@ac.upc.edu.

- J.M. Codina is with Intel Labs, Edifici Nexus-2, c./ Jordi Girona 29 (3A), Barcelona, Spain 08034. E-mail: josep.m.codina@intel.com.

- J. Sánchez and A. González are with the Intel-UPC Barcelona Research Center, Edifici Nexus-2, c./ Jordi Girona 29 (3A), Barcelona, Spain 08034. E-mail: \{jesus.sanchez, antonio.gonzalez\}@intel.com.

- D. Kaeli is with the Department of Electrical and Engineering, Northeastern University, 409 Dana Research Center, Boston, MA 02115. E-mail: kaeli@ece.neu.edu.

Manuscript received 4 Apr. 2008; revised 22 Aug. 2008; accepted 10 Sept. 2008; published online 11 Feb. 2009.

Recommended for acceptance by K. Ghose

For information on obtaining reprints of this article, please send e-mail to: tc@computer.org, and reference IEEECS Log Number TC-2008-04-0143.

Digital Object Identifier no. 10.1109/TC.2009.32.
One major task to be performed during instruction scheduling for clustered microarchitectures is cluster assignment. The performance of clustered processors depends heavily on the ability of the compiler to assign instructions to the appropriate cluster such that workload is balanced and intercluster communications are minimized.

In this work, we propose a Modulo Scheduling algorithm for clustered architectures. Modulo Scheduling is a commonly used technique to exploit parallelism in loops. The proposed heuristic-based algorithm (AGAMOS) generates schedules with high instruction-level parallelism while reducing register requirements and intercluster communication penalties. The technique is evaluated using 678 loops taken of the SpecFP95 benchmark suite. The execution time spent in these loops represents approximately 95 percent of the total execution time of these programs. The results presented in this paper show that for different cluster configurations, the proposed algorithm significantly outperforms previously proposed approaches.

Former proposals for code scheduling on clustered microarchitectures were based on a two-phase approach. First, instructions are assigned to clusters using just the information provided by the data dependence graph (DDG), and then the instructions are scheduled by strictly following the computed partition. More recent proposals have shown that performing cluster assignment and instruction scheduling in a single step can be more effective since the interaction between tasks can be taken into account. However, the drawback of these latter proposals is that the cluster assignment of each individual instruction is decided based on information about already scheduled instructions, which restricts assignment to use only a partial or local view of the dependence graph.

The technique proposed in this paper combines the benefits of global and local information to overcome the limitations of previous approaches [2]. The main feature of the technique is that the distribution of the instructions among the clusters is performed using a global view of the whole DDG, and at the same time, takes into account the main implications that the partition will have on the 
scheduling step. The final scheduling is performed as a separate phase, but the main interaction between these two tasks (e.g., required memory port usage, intercluster interconnect utilization, and register requirements) is precisely estimated during cluster assignment. The information considered is obtained through an estimate of the final schedule, which is called a pseudoschedule [3]. Our approach also features novel instruction replication heuristics to further reduce the number of communications [4], [5].

The remainder of this paper is organized as follows: Section 2 provides some background on modulo scheduling and graph partitioning techniques. Section 4 describes the proposed technique. Section 5 analyzes its performance. Section 6 reviews related work, and Section 7 summarizes this work.

\section{Modulo Scheduling}

Modulo Scheduling is an instruction scheduling technique for loops [32]. It achieves high performance by overlapping the execution of consecutive iterations. To begin the execution of a new iteration, it is not necessary that the previous iteration has finished its execution. A new iteration begins after a fixed number of cycles. This number of cycles is called the initiation interval $(I I)$. There is a lower bound for the $I I$, the minimum initiation interval (MII), which is computed taking into account resources (res $M I I)$ and recurrences $(\operatorname{rec} M I I): M I I=\max \{\operatorname{res} M I I, \operatorname{rec} M I I\}$.

If a single iteration takes it_length cycles to execute, then there can be up to $\lceil$ it_lengthII $\rceil$ iterations executing at the same time. This term is called the stage count $(S C)$. The stage count determines the length of the prolog and the epilog of the loop [33].

Using the terminology just defined, the time that a modulo-scheduled loop takes to complete execution is given by the following formula:

$$
T_{\text {exec }}=N_{\text {times }} \cdot I I \cdot\left(N_{\text {iters }}-1+S C\right),
$$

where $N_{\text {times }}$ stands for the number of times that the loop is executed and $N_{\text {iters }}$ represents the number of iterations of that loop. Hence, reducing the $I I$ is crucial to reducing $T_{\text {exec }}$.

The loop is represented as a DDG $G=(V, E)$, where $V$ stands for the set of nodes of $G$ (every node corresponds to an instruction in the loop) and $E$ stands for the set of edges of $G$ (every edge corresponds to a dependence between instructions in the loop).

Computing a schedule that minimizes the execution time for a given loop is an NP-complete problem. When using clustered architectures, the problem becomes even more difficult. In addition to scheduling all of the instructions in the loop, we have to schedule intercluster communications to pass values among clusters. In this work, we assume an intercluster connection network composed of a set of buses, as described in [35]. We will write n_buses to denote the number of buses in the network and lat_buses will stand for the latency of these buses.

Given a cluster assignment of the loop instructions, a communication is required whenever a value that is produced on one cluster has a consumer instruction located

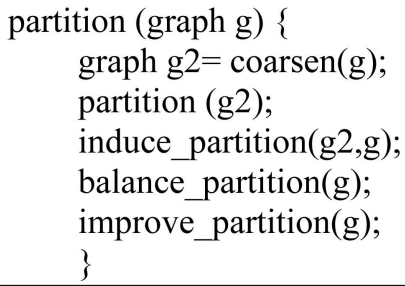

Fig. 1. Pseudocode for a multilevel partition algorithm.

in a different cluster. Let $n_{-}$coms stand for the number of communications induced by the cluster assignment. The minimum number of cycles required to schedule all these communications is referred to as the bus minimum initiation interval (busMII). It can be computed as follows:

$$
\text { bus MII }=\left\lceil\frac{n_{-} \text {coms }}{n_{-} \text {buses }}\right\rceil \cdot \text { lat_buses. }
$$

\section{Graph Partitioning}

Graph partitioning algorithms try to split the set of nodes of a graph into a predetermined number of parts, respecting some constraints regarding the number of nodes in each part, while trying to optimize some objective function.

There is a significant amount of previous work in the literature that discusses graph partitioning. It has been shown that multilevel algorithms are the most effective techniques [24]. These algorithms consist of two steps. In the first step, the graph is coarsened, that is, a graph with fewer nodes is generated by fusing pairs of adjacent nodes present in the original graph. Since the newly generated graph has fewer nodes, it is easier to partition. Hence, the new graph is partitioned, which induces a partition in the original graph. In the second step, a set of heuristics is applied in order to enhance the partition of the original graph. In Fig. 1, we present pseudocode for a recursive version of a multilevel partitioning algorithm. In the next sections, we describe the two steps in more detail.

\subsection{Multilevel Strategies}

\subsubsection{Coarsening}

Graph coarsening is an iterative process. At each step of this process, a graph with fewer nodes and fewer edges is obtained. Every new graph is built by fusing pairs of adjacent nodes of the graph produced by the previous step into a single macronode in the new graph. Fig. 2 shows an example. The original DDG is shown in the top left corner. In the next graph, down to the right, the groups of nodes that will be fused into a single macronode are marked in gray. ${ }^{1}$ In the next graph in the upper right, the three pairs of nodes marked in gray have been fused into three macronodes.

In order to decide which pairs of nodes will be fused into new macronodes, a matching is computed. A matching is a set of edges such that none of the edges in this set is adjacent. The pairs of nodes linked by edges belonging to the matching are fused into new macronodes. It is

1. The numbers beside the edges represent their weights, which are described in Section 3.2.1. 


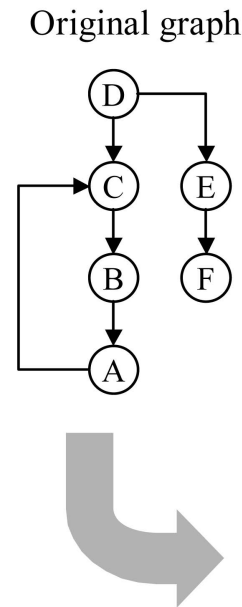

Find matching
Graph 2

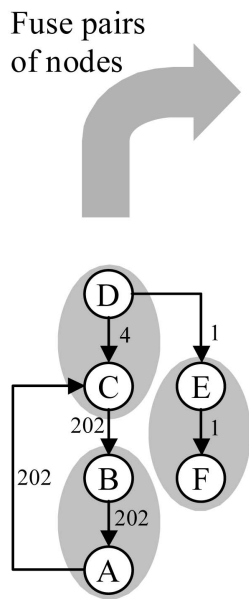

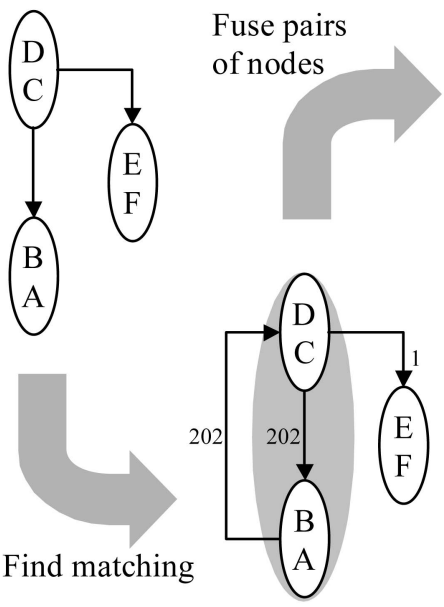

Graph 3

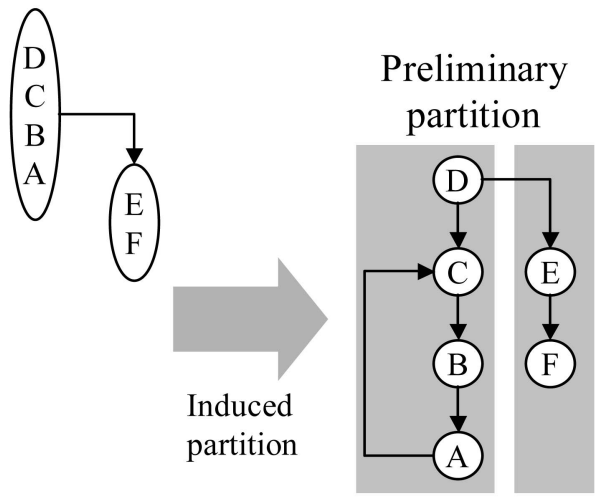

Fig. 2. Example of coarsening.

desirable that macronodes have a similar size and coarsening is performed simultaneously on the whole graph. Therefore, it is convenient that the matching contains as many edges as possible to coarsen all the parts of the graph at the same time. However, there may be edges that are better candidates than others to collapse. For this reason, each edge is given a weight and a maximum weight matching is computed. A maximum weight matching is a matching such that the sum of the weight of the edges belonging to it is the highest possible.

This process is repeated, producing a set of graphs. Every new graph has fewer, though coarser, nodes. In the example, three graphs are presented, the original one (five nodes), graph 2 ( three nodes), and graph 3 ( two nodes). Every node in the original graph belongs to only one node in the coarser graphs. For example, in Fig. 2, node $A$ in the original graph belongs to the macronode $A B$ in graph 2 and to the macronode $A B C D$ in graph 3.

Coarsening continues until we obtain a graph with a small number of nodes. This graph is partitioned using a straightforward process. Usually, coarsening stops when the number of nodes is equal to the number of partitions; the final graph is partitioned by assigning each macronode to a different partition. Since every node of the finer graphs belongs to one node of the final graph, the partition of the final graph also induces a partition in all the finer graphs. In particular, the partition in the final graph induces a partition in the original graph (referred to as the preliminary partition). In the example shown in Fig. 2, the graph is partitioned into two sets of nodes and coarsening stops when a graph with two macronodes (namely, $A B C D$ and $E F)$ is obtained. Then, each macronode is assigned to a different subset. Hence, we have obtained a partition in the original graph: nodes $A, B, C$, and $D$ are assigned to one subset, and nodes $E$ and $F$ to the other subset.

\subsubsection{Refining Heuristics}

Once the preliminary partition has been obtained, it is improved upon by analyzing the partitions induced in all the intermediate graphs produced during the coarsening process. The process starts with the result obtained in the final graph and revisits all intermediate graphs, until we reach the original graph. For this purpose, two heuristics are applied. These heuristics pursue different goals. The goal of the first heuristic is to keep the partition balanced (keeping balanced with respect to the number of nodes in each set of the partition). The goal of the second heuristic is to improve the partition (i.e., it tries to obtain a partition that improves the value of the objective function specified by the partition problem).

Several implementations have been proposed for both heuristics. Most of them are based on the well-known algorithm by Kernighan and Lin [25]. The general idea is to move nodes from one set of the partition to another whenever these movements produce a better partition (in terms of balancing or in terms of the value of the objective function).

\subsection{Graph Partitioning for Modulo Scheduling}

In this section, we explain a multilevel graph-partitioning algorithm for modulo scheduling. The main goal is to partition a loop in such a way that it can produce a highperformance schedule. To accomplish this purpose, the weights assigned to the edges to compute the matching for the coarsening and the heuristics used to improve the partition have been carefully designed.

Before describing both tasks in detail, we analyze the impact of clustering on performance. As we have seen in Section 2, the execution time of a modulo-scheduled loop is determined by the II and the length of the schedule. Splitting the instructions of the loop into clusters can impact both factors.

Clustering can impact the $I I$ in several ways. When the producer and the consumer of a given value are in different clusters, intercluster communication is needed. If the number of these communications is too high, the cluster interconnect may become a bottleneck and the $I I$ may need to be increased. Furthermore, adding a communication in a recurrence may increase the length of the recurrence, which, in turn, can impact the $I I$. Note that for the same reason, communications may also increase the length of the schedule. 
Another reason why clustering can impact the $I I$ is register pressure. Splitting instructions into clusters can increase register pressure due to many reasons. First, some values may be live in various clusters at the same time. Second, the workload is not the same in all clusters, so some of the workloads will have higher register requirements. In fact, an effective way of reducing the number of communications consists of placing as many instructions as possible in one (or a few) clusters. Therefore, this cluster would suffer from greater register pressure. Hence, reducing the number of communications and keeping register pressure low are two tightly correlated tasks.

In the next sections, we will show the actions taken during the partitioning step to address these issues. In particular, coarsening takes into account the structure of recurrences in order to avoid inserting communications where they may increase latency, whereas the heuristics used to improve the partition benefit from using a more global view of the structure of the graph that allows us to more accurately account for the impact of changing the number of intercluster communications and register pressure on the resulting $I I$.

\subsubsection{Weighting Edges to Coarsen the Graph}

As described in Section 1, coarsening fuses together pairs of adjacent nodes into a single macronode. The pairs of fused nodes will be placed in the same cluster in the preliminary partition. Hence, we want to fuse together nodes that are likely to belong to a common set of the partitions. The pairs to fuse are chosen by finding a maximum weight matching. Therefore, edges connecting nodes that are likely to belong to the same set of the partition should have bigger weights. In this section, we describe how we assign weights to edges.

Our final goal is to generate a partition that results in the fastest schedule. Hence, the weights have to guide us during partitioning so that we avoid increasing the $I I$ and the overall schedule length. As explained earlier, one reason for increasing the $I I$ is to introduce communications into recurrences. We will define weights that will try to avoid this situation by taking into account the structure of the recurrences. To accomplish this, the weight of an edge $e$, weight(e), needs to capture two different factors. The most important factor is the impact on execution time of adding a delay to this edge. We refer to this factor as $\operatorname{delay}(e)$. It is computed as follows:

$$
\begin{aligned}
\operatorname{delay}(e)= & \left(N_{\text {iters }}-1\right) \cdot(\text { newMII-MII }) \\
& + \text { new_longest_path }- \text { longest_path },
\end{aligned}
$$

where $N_{\text {iters }}$ is the iteration count of the loop (obtained through profiling), newMII is the minimum initiation interval (taking into account the latency of the communication), and longest_path and new_longest_path are the number of cycles of the longest paths in the graph before and after adding a delay in the edge, respectively.

Thus, the defined weights will be higher for edges between nodes that belong to a recurrence whose recMII could increase due to communications. Therefore, these recurrences will not be split. In addition, these weights also penalize edges where placing a communication would increase the length of the schedule.
As an example, we will show how to compute the delay for the edges of the graph of Fig. 2. Assume that all the instructions, and that communications between clusters, have a one-cycle latency. Assume also that the distance of the backward edge $\mathrm{A} \rightarrow \mathrm{C}$ is one. Then, the MII is determined by the recurrence $\mathrm{A}, \mathrm{B}, \mathrm{C}$ :

$$
M I I=\operatorname{rec} M I I=3 .
$$

The longest path in the graph is the path D, C, B, A. Since all the instructions have a one-cycle latency, the longest path is four cycles.

Let us see how to compute the delay for edge $C \rightarrow B$. If a communication was placed between these two instructions, the recurrence would be longer, which would increase the $I I$. Besides, the longest path would also be one cycle longer:

$$
\begin{gathered}
\text { newII }=4 ; \text { new_longest_path }=5 ; \\
\text { delay }=99(4-3)+5-4=100 .
\end{gathered}
$$

The delay is the same for the edges $\mathrm{B} \rightarrow \mathrm{A}$ and $\mathrm{A} \rightarrow \mathrm{C}$. Regarding edge $\mathrm{D} \rightarrow \mathrm{C}$, the delay is only one cycle (the $I I$ is the same), whereas the delay for edges $\mathrm{D} \rightarrow \mathrm{E}$ and $\mathrm{E} \rightarrow \mathrm{F}$ is zero.

The second factor that affects the weight of an edge is its slack, slack(e). The slack of an edge is defined as the number of delay cycles that could be added to this edge without affecting execution time. For some edges, adding a delay to them may not directly affect execution time. However, the effect of various communications may increase recMII or the length of the schedule. That is the reason why we also consider the slack in computing the weight.

For instance, increasing the latency of edges $\mathrm{C} \rightarrow \mathrm{B}, \mathrm{B} \rightarrow \mathrm{A}$, $A \rightarrow C$, and $D \rightarrow C$ causes a delay. Therefore, their slack is zero. On the other hand, increasing the latency of edge $\mathrm{D} \rightarrow \mathrm{E}$ or $\mathrm{E} \rightarrow \mathrm{F}$ does not affect execution time. However, if a communication was placed in both of them, then the length of the schedule would increase. Therefore, the slack is one cycle.

These two factors are converted into a single metric. The former factor, delay (e), is more important because it measures a direct impact on execution time whereas the slack measures only how close we are to increasing execution time. To ensure that any difference in the former factor has always a greater weight than the largest difference in the latter factor, we multiply the delay by the highest value of the slack plus one. Then, we add the highest value of the slack minus the actual slack (the lower the slack, the higher the weight). We finally add one unit, in order to avoid any zerovalued edge weights (because edges with zero weight will never be in the maximum weight matching). This is summarized by the following expression:

$$
\begin{aligned}
\text { weight }(e)= & \operatorname{delay}(e) \cdot\left(\max \_s l a c k+1\right) \\
& + \text { max_slack }-\operatorname{slack}(e)+1,
\end{aligned}
$$

where max_slack is the maximum slack of any edge of the graph.

Then, for instance, the weight of edge $\mathrm{C} \rightarrow \mathrm{B}$ is

$$
\text { weight }(C \rightarrow B)=100^{*} 2+1-0+1=202 .
$$

Coarsening is repeated until a graph with as many nodes as clusters in the target architecture is obtained. Then, each 
Preliminary partition

Cluster 1 Cluster 2
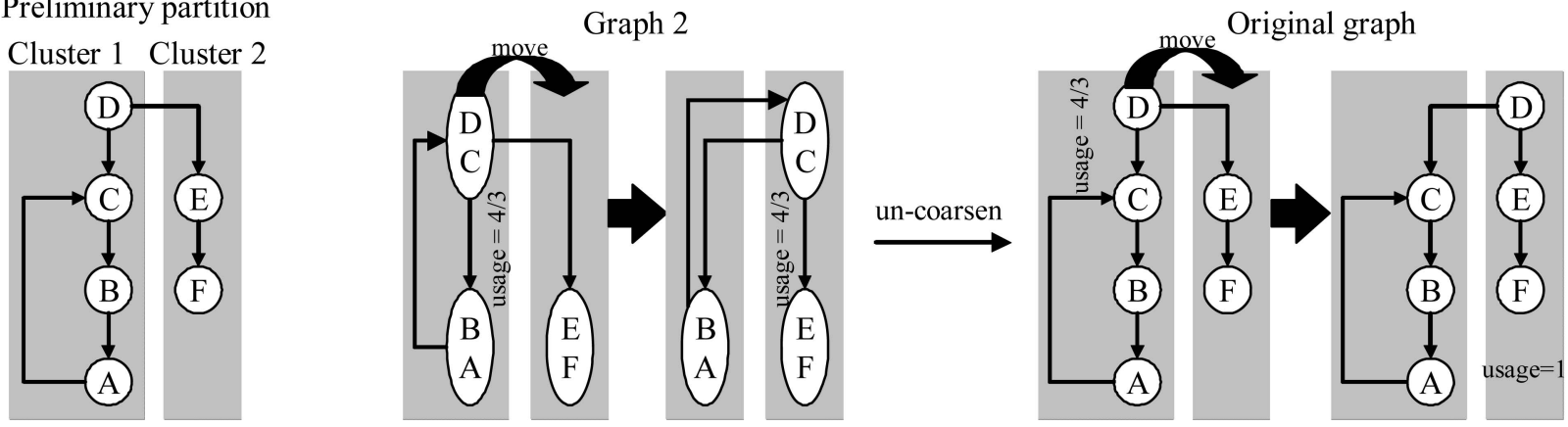

Fig. 3. Example for the balancing heuristic.

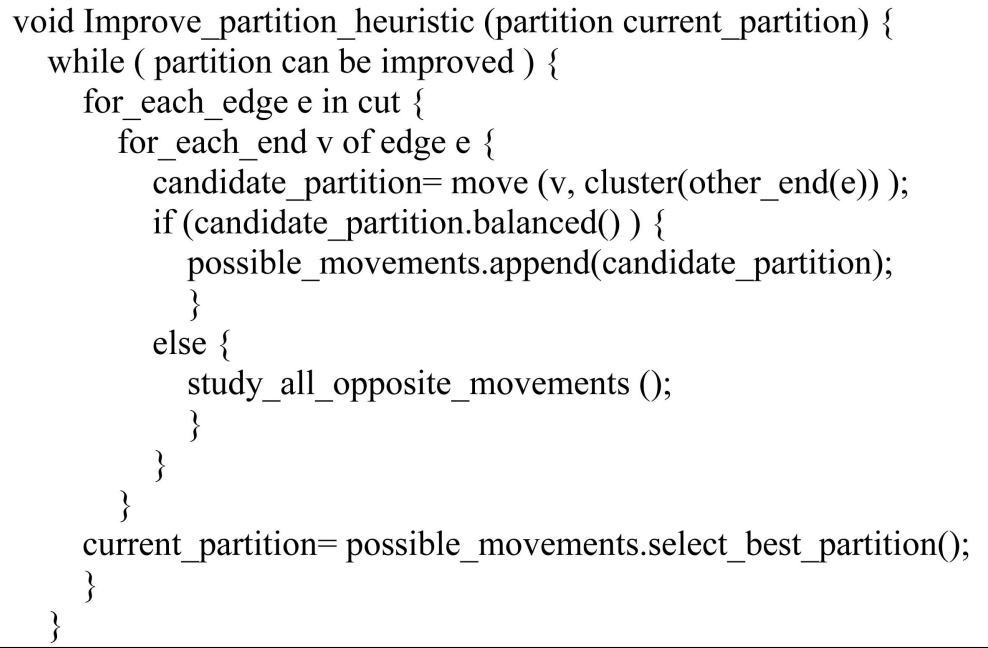

Fig. 4. Pseudocode for the Minimizing the Impact of the Partition on Execution Time heuristic.

node is assigned to a different cluster and this induces a preliminary partition of the original graph: every original node belongs to a unique macronode and it is assigned to the cluster where the macronode is. For instance, in Fig. 2, the induced preliminary partition puts instructions A, B, C, and $\mathrm{D}$ in one cluster and instructions $\mathrm{E}$ and $\mathrm{F}$ in the other.

\subsubsection{Refining the Partition}

In this section, we describe two heuristics used to improve the partition: one tries to balance the workload whereas the other tries to minimize the impact of the partition on execution time. Both heuristics are applied to all the intermediate graphs obtained during the coarsening process.

Improving workload balance. To have a valid partition for a given $I I$, each cluster must have enough resources to schedule its corresponding set of instructions. If this constraint is met, we will say that the partition is balanced. For instance, the left-hand side of Fig. 3 shows the partition obtained from the coarsening example shown in Fig. 2. If we assume one general-purpose functional unit per cluster and $I I=3$, then cluster 1 has insufficient resources to schedule the four instructions. In this case, we would say that the partition is unbalanced. On the other hand, if $I I=4$, the same partition would be balanced (even if the number of instructions in each cluster would not be the same).
In order to balance a partition, we apply a heuristic to all the intermediate graphs starting from the coarser ones. In Fig. 4, we present pseudocode for this heuristic.

At each step, the heuristic tries to improve workload balance if the current partition overloads any particular machine resource (i.e., its utilization factor is higher than 100 percent.) For this purpose, we analyze each resource, from most heavily loaded to the least loaded. For each cluster $C 1$ where this resource is overloaded, we try to make some balancing movements by applying the steps described in Fig. 4. In particular, for all nodes $v$ in the considered cluster $C 1$ containing any operation that uses the considered resource, we try to move that node $v$ from $C 1$ to any other cluster $C 2$. As long as $v$ is removed from $C 1$, the load in $C 1$ is reduced. On the other hand, the load in C2 increases. To consider this move as a candidate for balancing the partition, the resulting partition must not overload previously considered resources in C2. Besides, the resulting utilization of the currently considered resource in C2 must be lower than the previous utilization in $C 1$ (point 1 in Fig. 4).

In the example shown in Fig. 3, if we assume $I I=3$, then the usage of the FU in cluster 1 is $4 / 3$. Thus, the partition is not balanced and the balancing heuristic is applied. Moving nodes in graph 3 cannot provide any benefits in terms of balancing. We start with graph 2 . When moving any macronode of graph 2 from cluster 1 to cluster 2, the usage 

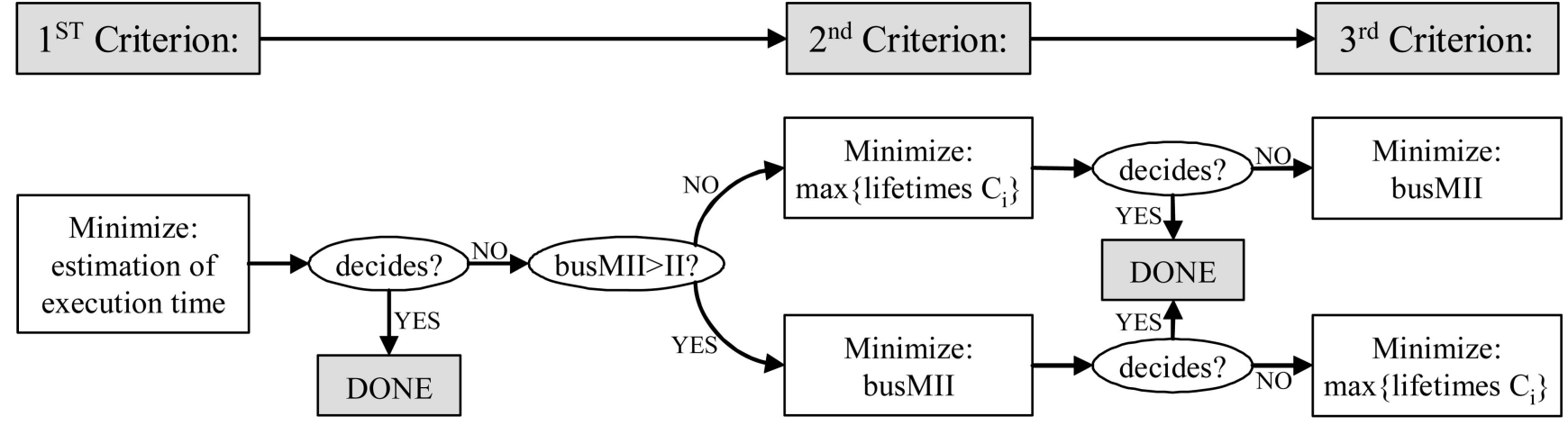

Fig. 5. Scheme of the decision criteria of the refinement heuristic.

of the FU in cluster 2 is $4 / 3$, which is the same as we had in cluster 1 before making the movement. Therefore, these movements do not provide any benefit. In the next iteration, we apply the heuristic to the original graph. When we move any of the nodes of cluster 1 to cluster 2, we obtain a balanced partition. All the movements provide the same benefit in terms of balancing the partition. However, it is intuitively clear that the best option is to move node $D$, as shown in Fig. 3, since the other movements introduce unnecessary communications. Hence, a way to choose this movement is needed, instead of the other choices which are less beneficial for performance.

When several movements achieve the same degree of balancing, we choose among them (point 2 in Fig. 4) with the same criteria used by the heuristic that minimizes the impact of the partition on performance (we describe this heuristic in the next section). Once a movement has been selected, the whole process is repeated until no resource is overloaded or until no beneficial movement can be found.

Minimizing the impact of the partition on execution time. For each graph generated during the coarsening process, from the coarsest to the finest, after applying the improving workload balance heuristic, we try to reduce the impact of the partition on execution time. To achieve this goal, we use an iterative heuristic. Its pseudocode is shown in Fig. 4.

The heuristic generates different partitions and chooses the best one (i.e., the partition that is likely to generate the fastest schedule) until no further improvement can be achieved. Thus, we need a strategy to generate different partitions and a way to compare them.

Different partitions are generated by moving nodes from one cluster to another. Since the entire partition space cannot be explored, we need to select the movements that are more likely to improve the partition. Since intercluster communications are a major cause of $I I$ increase, we only consider moving a node $v$ if that node $v$ is adjacent to an edge belonging to the cut of the partition (i.e., a node $v$ that has a neighbor in a different cluster). In particular, we consider moving $v$ from its current cluster $C 1$ to each cluster $C 2 \neq C 1$, where it has an adjacent node (point 1 in Fig. 4). If there are not enough free resources in C2 to place node $v$ there, but the required resources can be made available by moving a node from $C 2$ to $C 1$, all feasible exchanges between pairs of nodes are considered (point 2 in Fig. 4).

Among all the generated partitions, we want to choose the one that is most likely to produce the best schedule (point 3 in Fig. 4). The most rigorous approach would be to compute the schedule associated with each partition and select the best one. Obviously, this would be too timeconsuming. However, we can compute an approximate schedule that we call pseudoschedule to accurately estimate all the factors that can influence the execution time of the final schedule. In particular, the pseudoschedule is used to estimate the $I I$ and the $S C$ of the partition and the number of lifetimes used in each cluster. The way to compute the pseudoschedule is described in Section 3.3.

Then, for all possible partitions generated by only moving a single node or swapping a pair of nodes, we select a partition according to the criteria presented in Fig. 5. First, we choose the partition with the shortest estimated execution time. In case of a tie in this metric, we select the partition that is most likely to be improved in future refinements. In particular, we try to reduce the load on resources that are most likely to cause the $I I$ to increase (i.e., intercluster buses and registers). Hence, if busMII $\leq I I$, then we assume that the number of communications is already sufficiently low. Therefore, in the case of a tie in the estimated execution time, we choose the partition that minimizes register pressure. More specifically, we choose the partition that further reduces the maximum number of lifetimes used in a cluster. Alternatively, if busMII $>I I$, this indicates that communications are critical and we select the partition that minimizes busMII.

In the case of a tie in the second metric (whether we are considering registers or communications), we select the partition that minimizes the other criterion (i.e., if we used register pressure as the second metric, then we use communications as the third; if we used communications as the second metric, we use register pressure as the third).

Once the best partition is found, the node movement (or the exchange of node pairs) that produced the selected partition is applied. This process is repeated until no further benefit can be obtained.

\subsection{Pseudoschedules}

When comparing two partitions, it is difficult to decide which one could produce a better schedule because there are multiple constraints that cannot be quantified at the partitioning step that influence the quality of the final schedule, like the length of the schedule and the register pressure. To properly estimate the impact of these factors on the final schedule, we build an approximate schedule: the pseudoschedule. 
To produce a pseudoschedule, we first compute a lower bound on the $I I\left(l b_{-} I I\right)$ as follows:

$$
l b \_I I=\max \{I I, \text { new } \operatorname{Rec} M I I, \text { bus } M I I\},
$$

where $I I$ is the initiation interval for which we are trying to produce a schedule, newRecMII is computed as the recMII while taking into account the latency of the necessary communications and busMII is the bus minimum initiation interval described in Section 2.

Next, we assume that the initiation interval of the pseudoschedule $\left(p s_{-} I I\right)$ is equal to this lower bound $\left(p s_{-} I I:=l b_{-} I I\right)$, and then try to find a suitable slot for each node. Since the pseudoschedule needs to be computed as accurately as possible, nodes are scheduled using the same rules as those used by the final scheduler. Therefore, according to the ordering described in [28], each node is scheduled as close as possible to its predecessors/successors in order to shorten lifetimes. Unlike the final scheduler, if we cannot find a slot available to schedule an instruction in the cluster to which it belongs, we assign that node to a given cycle, even if there are insufficient resources available in this cycle.

We determine this cycle as follows:

1. If the node $v$ has only predecessors in the partial pseudoschedule: $\operatorname{cycle}(v)=\operatorname{EarliestStart}(v)+I I$.

2. If the node has only successors in the partial pseudoschedule: $\operatorname{cycle}(v)=$ LatestStart $(v)-I I$.

3 . If the node has both predecessors and successors in the partial pseudoschedule: $\operatorname{cycle}(v)=$ $[$ LatestStart $(v)-$ EarliestStart $(v)] / 2$.

By computing cycles in this way, we penalize a partition by extending lifetimes and increasing the length of the schedule. This penalty is relatively small since this is just an intermediate partition that can still be improved in later steps. For case 3, if the node has both predecessors and successors in the partial pseudoschedule, it means that the node represents the last instruction in a recurrence loop (see [28]), and that some dependences cannot be met. In this case, a larger penalty is assessed to the $p s_{-} I I$. This recurrence may have to be split. Since splitting a recurrence generally incurs two communications, we penalize the ps_II by more than twice the bus latency

$$
p s \_I I=l b \_I I+2 \cdot l_{a} \_ \text {buses }+1 \text {. }
$$

Note that for this approximate schedule, an unlimited number of registers is assumed. However, once the pseudoschedule is built, the number of lifetimes it requires, as well as the maximum number of lifetimes that overlap, is computed to provide more information to the partition.

Iteratively applying this algorithm, all the nodes in the graph are pseudoscheduled (i.e., assigned to a cycle). The algorithm investigates no more than $l b \_I I$ different positions. Thus, the time complexity to produce a pseudoschedule is linear with $I I \cdot|V|$.

The computed pseudoschedule is quite accurate, especially when the partition is balanced and there is enough space to schedule all instructions in the cluster as specified by the partition. Therefore, moving nodes among clusters during the partitioning step can target different goals, depending on the most constraining factors. For instance, a goal can be to reduce the number of communications, evenly distributing register pressure, to produce a better distribution of recurrences among clusters or reduce the length of the schedule.

\section{Proposed MS Scheme}

Several heuristics have been proposed to modulo schedule loops [15]. These heuristics try, in general, to maximize ILP and some of them also try to minimize register pressure. For clustered architectures, modulo scheduling is more complex since it has also to deal with intercluster communications and the distribution of the workload among clusters.

In this work, we propose $A G A M O S$, a new technique that performs cluster assignment using a multilevel graphpartitioning scheme. In this way, the scheduler uses global information of the DDG and it can achieve a better workload balance at the same time that it can reduce the number of communications. The generation of this partition is guided by approximate schedules, called pseudoschedules, which provide very accurate information about the properties of the final schedule. During this process, selected instructions are replicated in order to reduce the number of communications. The scheduler tries to follow the cluster assignment produced by the graph partition. However, the scheduler can refine this assignment.

In this section, we provide an overview of the code generation algorithm, the replication heuristics, and the scheduler.

\subsection{Overview of the Scheduling Algorithm}

In Fig. 7, the high-level flow of the proposed algorithm is shown. The scheme tries to find a valid schedule that minimizes the $I I$. It starts with $I I=M I I$. If it fails, the $I I$ is increased by one unit.

To produce a feasible schedule for the current $I I$, the DDG is partitioned according to the algorithm we have described in Section 3.2. The partitioning technique includes a set of heuristics to replicate instructions in various clusters in order to reduce the number of communications, as described in Section 4.2.

The last step of the algorithm is the scheduling phase (Section 4.3). Each instruction is scheduled in the cluster, where it has been assigned by the partition. If the algorithm succeeds and a valid slot is found, then scheduling proceeds to the next instruction. If a valid slot cannot be found for the current instruction in the cluster where it has been assigned by the partition, it is tried to be scheduled in other clusters. If a suitable cluster is found, the instruction is scheduled in that cluster and the algorithm continues with the next operation (starting again with the cluster dictated by the partition). If we reach a situation where an instruction cannot be scheduled in any cluster, the process stops, the $I I$ is increased, and the described process restarts.

\subsection{Instruction Replication}

At each step of the partitioning process, before computing the pseudoschedule, we compute the busMII. If bus $M I I>I I$, this means that intercluster communication bandwidth is insufficient to schedule all the communications. For example, the graph shown in the left-hand side of Fig. 6 represents a DDG. This graph is partitioned into four sets of nodes and each set is assigned to a different cluster: 

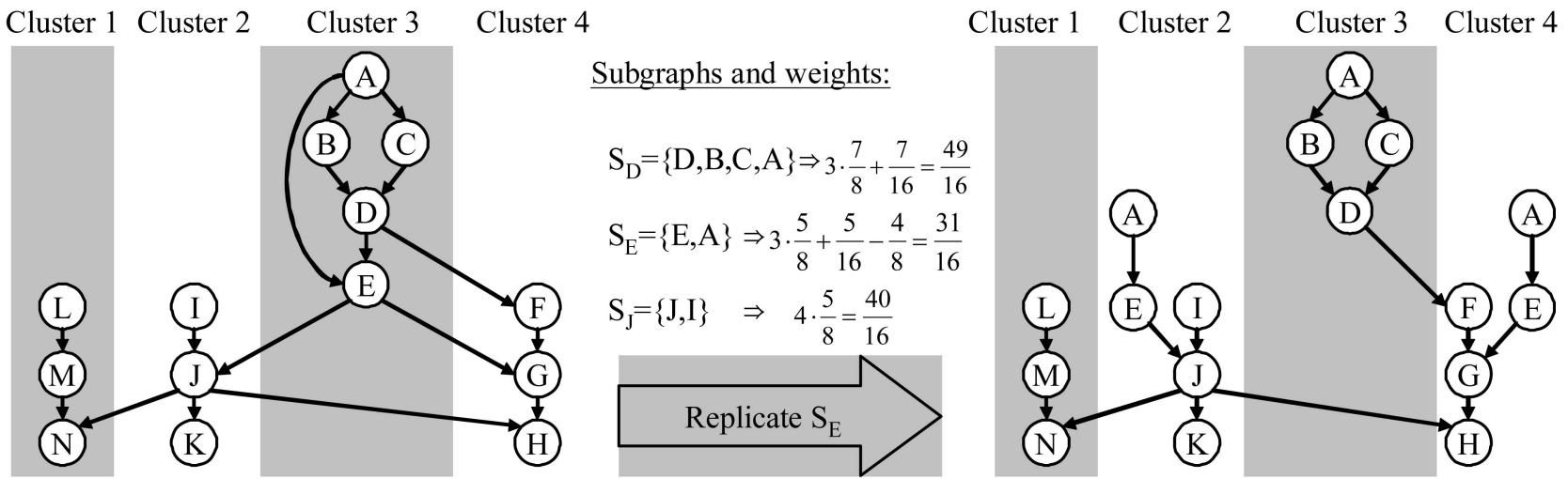

Fig. 6. Example of instruction replication to reduce communications.

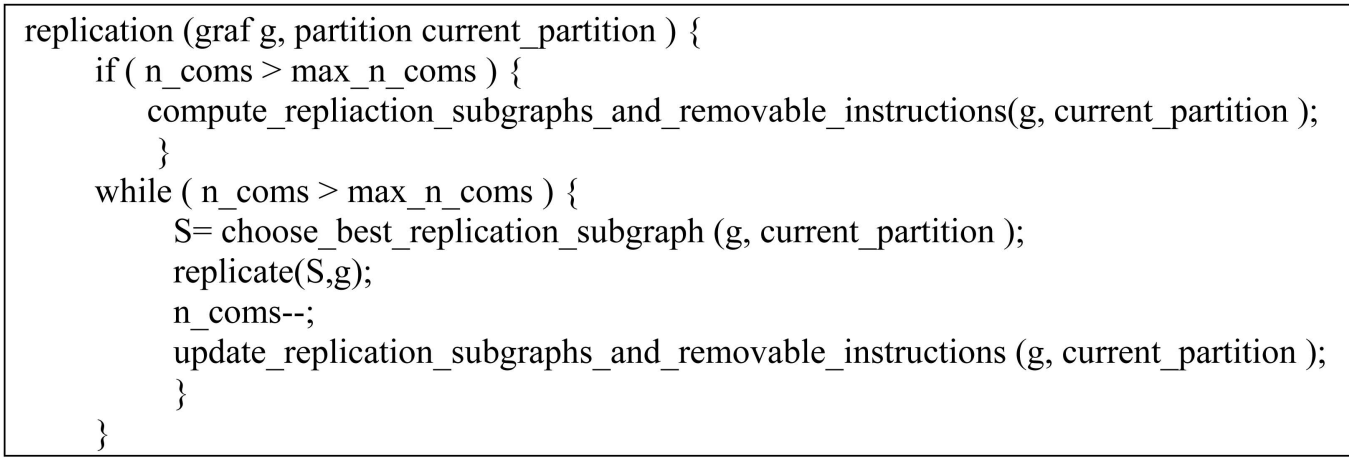

Fig. 7. Pseudocode for the replication heuristic.

$\{L, M, N\}$ to cluster $1 ;\{I, J, K\}$ to cluster 2; $\{A, B, C, D, E\}$ to cluster 3; and $\{F, G, H\}$ to cluster 4 . In the resulting partition, there are three values that have to be communicated: the values produced by instructions $D, E$, and $J$. If we assume an $I I=2$ and an intercluster communication network with one bus that has one-cycle latency (recall that we assume intercluster networks that broadcast the values to all clusters), then busMII $=3>I I$. In this case, there is an excess of communication.

We refer to the maximum number of communications that can be scheduled for the current $I I$ as the max_n_coms, and to the number of excess communications as the extra_coms. The number of excess communications can be computed as follows:

$$
\begin{array}{r}
\text { extra_coms }=n \_c o m s-\max \_n \_c o m s \\
\max \_n \_c o m s=\lfloor I I / \text { lat_buses }\rfloor \cdot n \_b u s e s,
\end{array}
$$

where $n_{-}$coms stands for the number of communications implied by the partition, as defined in Section 2 .

The number of communications can be reduced (and so can extra_coms) by replicating selected instructions in specific clusters. In this section, we describe a heuristic to achieve this purpose.

\subsubsection{Replication Subgraphs}

The replication subgraph corresponding to an instruction com, whose resulting output value has to be communicated to other clusters, is the minimum set of nodes that have to be replicated in order to remove that communication. We will denote this subgraph as $S_{\text {com }}$.
A simple example is presented in Fig. 6. The replication subgraph corresponding to the communication of the value produced by instruction $D$ has four nodes: $S_{D}=\{D, B, C, A\}$; the replication subgraph for $\mathrm{E}$ is: $S_{E}=\{E, A\}$. Node D does not belong to $S_{E}$ because it is unnecessary to replicate $\mathrm{D}$ to remove communication $\mathrm{E}$ since the value produced by D is already communicated and is available in the other clusters. The replication subgraph of instruction J is: $S_{J}=\{J, I\}$.

\subsubsection{Removing Unnecessary Instructions}

After removing a communication by replicating instructions in other clusters, there may be some original (nonreplicated) instructions that are no longer needed. An example can be found in Fig. 6. The graph in the right-hand side of the Figure represents the resulting DDG after removing the communication of node $E$ by replicating $S_{E}$ in clusters 2 and 4 . Then, the original instruction $E$ in cluster 3 is not needed. The value that it produces is not used by any other instruction. The two successors of $E$ ( $J$ and $G$ ) obtain their copy of $E$ from the copy generated in their respective clusters. Therefore, the original instruction $E$ can be removed from the graph, so more resources will become available in cluster 3 . Removable instructions can be anticipated before replication. Thus, they can also be taken into account when selecting which subgraph to replicate.

\subsubsection{Replication Heuristic}

In Fig. 7, pseudocode for the replication heuristic is presented. It works as follows: first, we compute the replication subgraphs and identify the removable instructions for all of the values that need to be communicated. 
Then, we choose the best candidate for replication and replicate it. After performing replication, the number of communications ( $n_{-}$coms) is reduced by one, and the number of excess communications (extra_coms) is also reduced by one. This process is repeated until the number of communications becomes equal to the maximum number of communications allowed for the current $I I$ $\left(n_{-} c o m s=m a x_{-} n_{-} c o m s\right)$ and the number of excess communications is zero (extra_coms $=0$ ), or until no further replication is possible due to resource constraints.

In order to select the best candidates for replication, we take into account the usage of resources. In some cases, the number of extra_coms is large, so there may be insufficient resources to replicate all the necessary instructions. Therefore, it is important to reduce the number of extra instructions that need to be added. Furthermore, reducing the number of extra instructions is also beneficial for other reasons such as register pressure, energy consumption, and code length. Hence, our metric is based on reducing the number of extra instructions.

To determine the weight of a subgraph, we first assign weights to the nodes that have to be copied to other clusters to avoid the communication and the nodes that can be removed after the subgraph has been replicated. Then, the weight of the subgraph is the sum of the weights of the nodes that have to be replicated, minus the weight of the nodes that can be removed.

To compute the weight of a single node $v$, we take into account the degree to which resources that are used by the instruction will be constrained if the subgraph is replicated. If a node belongs to more than one subgraph, it can be replicated and then used multiple times

$$
\text { weight }(v, c)=\frac{\frac{\text { usage }(\text { res, }, c)+\text { extra_ops (res,c,subgraph })}{\text { available(res,c) } \cdot I}}{\left|\left\{S_{C} / v \in S_{C}\right\}\right|},
$$

where usage $($ res, $c)$ stands for the number of instructions that use resource res and that are assigned to cluster $c$ for the given partition; extra_ops(res, c, subgraph) represents the number of instructions that use resource res that have to be replicated in cluster $c$ in order to replicate the subgraph, and finally, available $($ res,c) is the number of resources of type res in cluster $c$.

\subsection{Final Schedule}

When the partition has been completed, the final schedule is computed. In this section, we present the approach used to perform instruction scheduling and register allocation. Both tasks are carried out at the same time, generating spill code on the fly when needed. The approach is based on the URACAM modulo scheduling framework for clustered VLIW architectures [14].

\subsubsection{Instruction Scheduling}

First of all, the nodes of the DDG are sorted according to the Swing Modulo Scheduler [28]. Then, following this ordering, the schedule is produced through an iterative process that works by adding instructions to a partial schedule until all instructions have been scheduled. Each instruction is scheduled in the cluster assigned during the partition.

Once a new partial schedule has been obtained, the proposed technique includes mechanisms in order to reduce the pressure on a given type of resource at the expense of increasing the pressure on others. This is achieved by applying some transformations to the partial schedule:

1. Reduce register pressure by inserting spill code.

2. Reduce the bus pressure by performing communications through memory. Communications through buses can be removed by storing the value from the source cluster to a given memory location and loading it in the clusters from memory where it is needed.

Both of these transformations increase the pressure on memory ports. If later in the schedule memory pressure becomes too high, we can reduce it by undoing any of the previous transformations (i.e., by either removing spill code or inserting communication instructions that use the interconnection network instead of memory).

For each partial schedule, all transformations are applied. For each transformation applied, a new partial schedule is obtained. Therefore, we need a mechanism to compare the partial schedules and decide which one is the best. For this purpose, we use the figure of merit described in Section 4.3.2). Transformations are applied until no further improvement (in terms of the figure of merit) can be achieved.

If no feasible schedule can be found for a given instruction following the cluster assignment of the partition, then we try to place that instruction in the other clusters. In turn, these alternative schedules are improved by means of the transformations. Note that the original URACAM proposal did not use graph partitioning to perform cluster assignment. Thus, in contrast to our approach, URACAM tried to schedule each node to each cluster.

If no feasible schedule is found either, then the $I I$ is increased. Then, the partition and replication are redone in order to take advantage of the bigger amount of resources available and the scheduling process is restarted.

\subsubsection{Figure of Merit}

When different partial schedules are suitable, we need a function that allows us to compare them and select which one is better. We define a function that we will refer to as the figure of merit.

The figure of merit is based on the utilization of the most critical resources. The underlying assumption is that the more constrained a resource, the more likely to impact execution time.

The utilization of functional units is determined beforehand and does not depend on the schedule. The selected II has been chosen in such a way that there are enough slots for any required functional unit operation. However, the utilization of other resources is unpredictable and depends on the particular schedule. These critical resources are the intercluster connection network, the memory ports, and the registers.

Given a partial schedule and the current instruction that is to be scheduled, we use a multidimensional figure of merit to compare the different partial schedules resulting from inserting the instruction in alternative slots. The figure of merit consists of a set of $\left(2 \cdot n_{-} c l s t+1\right)$ percentages:

1. One for intercluster communications-the percentage of free communication slots (before scheduling 
the current instruction) that are consumed by the newly inserted instruction.

2. n_clust for memory-for every cluster, the percentage of free memory access slots (before scheduling the current instruction) that are consumed by the newly inserted instruction.

3. n_clust for registers-for every cluster, percentage of free lifetimes (before scheduling the current instruction) that are consumed by the newly inserted instruction.

The reason why we use the percentage of remaining resources that are consumed by the analyzed instruction is that scarce resources are more valuable than abundant ones. More specifically, the value of a given type of resources is inversely proportional to the amount of currently remaining resources of this type.

Then, we need a function that compares two figures of merit and determines which one is better. For this purpose, the components of each figure of merit are sorted from highest to lowest. Then, values are compared pairwise, starting from the highest value until a significant gap is found (greater than a given threshold ${ }^{2}$ ). In this case, the figure of merit with the lowest component is chosen. If all pairs of components are similar, a choice is made by summing the components for each figure of merit and selecting the one with the lowest sum.

This approach of comparing figures chooses the one that maximizes the available resources of the most heavily used type of resource. This heuristic can be characterized as trying to benefit the most constrained (most used) resource so that the difference between the least constrained (least used) resource and the most constrained is gradually reduced.

In contrast to the original URACAM approach, the partition provides additional information about the usage of resources (i.e., usage of memory ports in each cluster). This additional information is taken into account when computing the figure of merit that results in a better estimation of the impact of the decisions taken at scheduling time.

\section{Evaluation}

\subsection{Experimental Framework}

Our Modulo Scheduling algorithm has been implemented in the ICTINEO compiler [8]. For the evaluation, we used all the loops in the SPECfp95 benchmark suite that have neither subroutine calls nor conditional exits. Loops with conditional structures in their bodies have been IFconverted [7] into a single basic block loop. In addition, we used profiling to obtain the number of times and the average number of iterations that the loops were executed. The loops that executed, on average, less than four iterations were discarded. In total, we used 678 loops that accounted for more than 95 percent of the total number of executed instructions. Loop unrolling was disabled in order to prevent the increase in code size, which is critical for these processors.

We study three different clustered VLIW designs. Each is 12-issue and has the same number of total resources that are divided homogeneously across the different clusters. These clustered designs are shown in Table 1.

2. Based on the original URACAM technique [13, we set the threshold to 10 percent for the experiments reported.
TABLE 1

Clustered VLIW Designs and Latency of the Instructions

\begin{tabular}{|c|c|c|c|}
\hline Resources & Unified & 2-cluster & 4-cluster \\
\hline INT/cluster & 4 & 2 & 1 \\
\hline FP/cluster & 4 & 2 & 1 \\
\hline MEM/cluster & 4 & 2 & 1 \\
\hline
\end{tabular}

\begin{tabular}{|c|c|c|}
\hline Latencies & INT & FP \\
\hline ARITH & 1 & 3 \\
\hline MUL/ABS & 2 & 6 \\
\hline DIV/SQRT & 6 & 18 \\
\hline MEM & $2 / 3$ & $2 / 3$ \\
\hline
\end{tabular}

We will refer to the first design as unified. This architecture is composed of a single cluster with four functional units of each type (integer, floating point, and memory) and a unique register file. The 2-cluster design has two functional units of each type and half the number of the registers per cluster, whereas the 2-cluster design has one functional unit of each type and a quarter of the number of registers per cluster.

For the 2 and 4-cluster designs, different configurations based on the number of registers, number of buses, and latency of the buses are considered. Each configuration is identified as a sequence of letters and numbers, $N \mathrm{c} M \mathrm{~b} P \mathrm{l} Q \mathrm{r}$, where $N$ stands for the number of clusters, $M$ represents the number of buses, $P$ stands for the latency of these buses, and $Q$ represents the number of registers.

For all configurations, the memory hierarchy is shared by all the clusters and considered perfect (i.e., all accesses hit in cache). The latency assumed for hits is two cycles for the configurations whose bus latency is one cycle and three cycles for the configurations whose bus latency is two cycles. The latency of the remaining instructions is presented in Table 1.

The unified design has the same resources as the 2 and 4-cluster designs. However, for a unified architecture, register pressure is lower. Moreover, a unified architecture does not suffer from intercluster communication penalties. Therefore, the instructions per cycle (IPC) measure for the unified configuration is an upper bound on what can be achieved by the clustered architectures. Note that IPC is independent of the processor cycle time. The clustered organizations will certainly benefit from a faster clock, and thus, an IPC for a clustered configuration that approaches the IPC obtained by a unified architecture translates to an overall performance improvement when the cycle time is considered.

In this section, we use IPC as the main performance metric. The IPC includes the contribution of the prolog and epilog. Only the original loop instructions are considered, i.e., IPC does include neither replicated instructions, nor communications nor spill instructions.

For some loops, their associated initiation interval reaches a limit that makes modulo scheduling inappropriate. In this case, list scheduling is applied. Nevertheless, we have noticed that this case occurs for just a few loops in our suite.

\subsection{Performance Figures}

Fig. 8 shows the results for different cluster configurations. In this figure, we show IPC for 2-cluster architectures that have one bus, one-cycle latency; 4-clusters with one bus with a one-cycle latency, and 4-clusters with one bus with a two-cycle latency. The graphs on the left-hand side of Fig. 8 correspond to architectures possessing 32 registers, whereas 

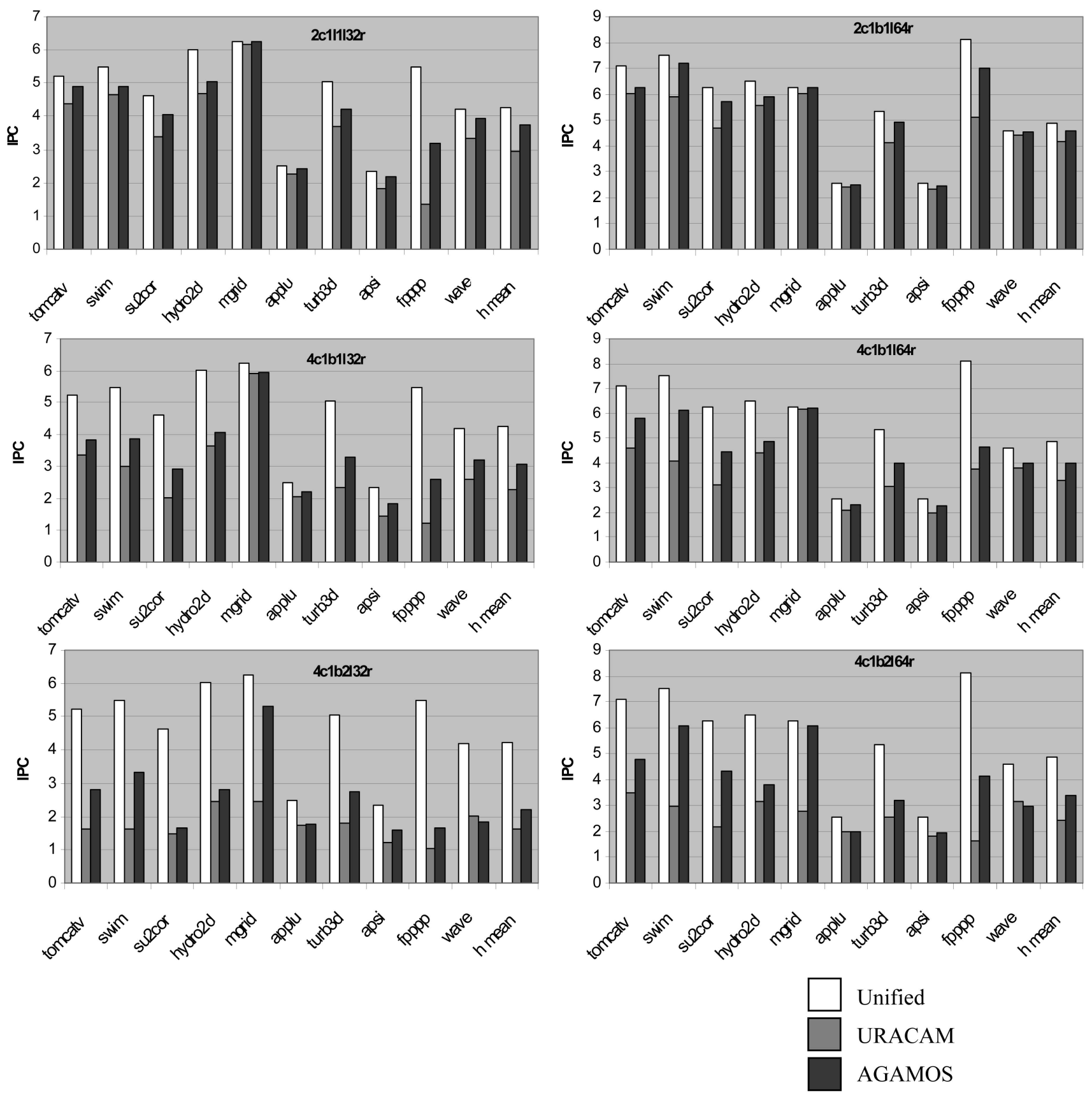

Fig. 8. IPC for the different configurations.

the graphs on the right-hand side correspond to architectures with 64 registers.

We plot results for three different approaches. The white bar represents the unified configuration. The gray bar represents URACAM. We have chosen URACAM as a baseline for comparison because it is a state-of-the-art scheduler that performs cluster assignment, instruction scheduling, and register allocation in a single step. This family of schedulers has been shown to outperform previous proposals, as will be further discussed in Section 6 . Finally, the black bar plots IPC for the approach described in this paper, AGAMOS.

The main conclusion that we can draw from these figures is that the scheme presented in this work produces significant gains for all configurations and all programs

with respect to URACAM. On average, the schedules produced by the proposed techniques improve IPC from 10 percent up to 40 percent over URACAM. The smallest speedup (10 percent) is achieved for the $2 \mathrm{c} 1 \mathrm{~b} 1164 \mathrm{r}$ configuration. This is due to the fact that this architecture is the least constrained of the architectures considered, and so the performance achieved is close to that of a unified architecture. Therefore, there are fewer opportunities for improving the workload balance and reducing the number of communications.

On the other hand, the most constrained configuration, i.e., 4c1b2132r receives the greatest benefits from using replication and pseudoscheduling. In this case, the speedup is close to 40 percent. This is due to the fact that for this 


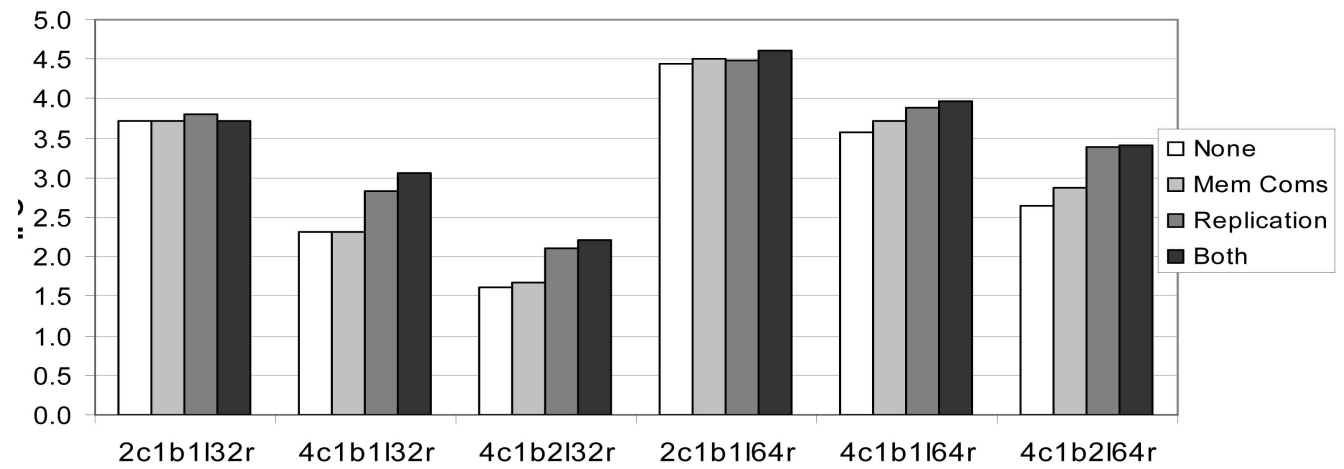

Fig. 9. IPC achieved using the proposed techniques to reduce the number of communications.

particular architecture, it is critical to evenly balance workload. Both communications and register pressure are critical. Therefore, reducing communications at the expense of placing many instructions in a single cluster is not a good strategy. Our pseudoschedules take this fact into account. In addition, replication provides an alternative way of reducing the number of communications.

If we look at the IPC results in more detail, we can see that for some programs (e.g., swim or fpppp for 4c1b2larchitectures), with both 32 and 64 registers, the speedup is over 100 percent. In addition, there are many programs such as tomcatv or su2cor for which the speedup is over 50 percent. We have observed that the programs with the higher speedups are dominated by loops with a high trip count. For these loops, the $I I$ has a big impact on execution time. AGAMOS is very efficient at reducing the $I I$ because it can solve different problems such as communications in recurrence circuits, the excess of communications, or highregister pressure.

On the other hand, for programs such as apsi or applu, the speedup achieved is much lower. This is due to the fact that these programs have some dominating loops with a low trip count. For these loops, the length of the schedule is as important as the II. AGAMOS takes also into account the length of the schedule but the potential to reduce it is smaller because it is limited by the longest paths in the $D D G$. Hence, the speedups obtained are lower.

Finally, the speedups for mgrid are low for some configurations and very high for the most restrictive ones. Note that when the speedup is low, the IPC is close to that of the unified architecture. Hence, AGAMOS has no potential to increase performance for this program and these configurations. When the architecture is more restrictive, AGAMOS can find a good schedule.

\subsection{Reducing the Number of Communications}

In this section, we evaluate the effect of the techniques used to reduce the number of communications, that is, communications through memory and instruction replication. In Fig. 9, we can see the average IPC obtained for the SPECfp95 programs with the proposed scheduling scheme for the different possible alternatives. We compare a baseline that uses the partitioning technique guided by pseudoschedules but does not consider communications through memory and does not perform instruction replication (white bars that correspond to the work presented in
[3]), with scheduling only communications through memory (light gray bars), with using only instruction replication (dark gray bars), or with using both techniques (black bars).

The main conclusion is that both techniques are useful to achieve a better IPC. Obviously, this is due to a reduction in the number of communications, which is often the bottleneck for cluster scheduling. Therefore, the more constrained the interconnection network, the larger the benefit. Hence, the biggest benefits are obtained for the architectures supplied with two-cycle latency interconnection networks. For example, for the two-cycle latency bus configurations, the speedup achieved when using both techniques is greater than 30 percent.

Another important conclusion is that replicating instructions provides greater IPC benefits than does scheduling communications through memory (dark gray bar and light gray bar, respectively). This is due to the fact that instruction replication can use all free resources, whereas memory communications benefit only from memory slots. Besides, scheduling communications through memory reduces the chances to schedule spill code. This fact is especially significant when register pressure is high. As we can see, for configurations with 32 registers, the performance obtained by a scheduler that pays no attention to reducing the number of communications is very close to the performance obtained from a scheduler that schedules communication through memory.

\section{Related Work}

Finding an optimal schedule in a resource-constrained environment is well known to be NP-complete. Many heuristics have been proposed in order to find near-optimal schedules. These heuristics have different goals: increasing throughput [23], [33], minimizing register pressure [16], reducing the effect of cache misses, or improving many of them simultaneously [28], [34]. All of these studies focus on modulo scheduling algorithms targeting unified (i.e., nonpartitioned) architectures.

There have been a number of modulo scheduling approaches proposed for clustered architectures. Nystrom and Eichenberger [29] performed cluster assignment and instruction scheduling in two independent steps. Cluster assignment was done using a straightforward algorithm based on the structure of the graph. It required less compilation time than multilevel strategies but it was not able to sufficiently reduce the number of communications. 
In fact, this scheme was outperformed by Sánchez and González [35], who proposed to integrate cluster assignment and instruction scheduling. Fernandes et al. [18] also perform cluster assignment and instruction scheduling in a single step. However, they assume a different architecture with an unusual register file organization based on a set of local queues for each cluster and a queue file for each communication channel. Finally, also Hiser et al. [21] presented a two-step approach in which a greedy algorithm is used for partitioning software pipelined loops.

More recent approaches [14], [37] perform cluster assignment, instruction scheduling and register allocation in a single step. These algorithms are the state-of-the-art and we use them as our baseline.

In recent works, some extensions to modulo scheduling have been proposed in order to deal with heterogeneous clustered VLIW microarchitectures where each cluster can run at a different frequency and voltage [6].

There are also several works on acyclic scheduling for clustered architectures ([10], [30]). Probably, the closest one to our technique is [13], where graph partitioning is also used to perform cluster assignment.

There is limited prior work related to instruction replication. Chaitin et al. [11], in their work on register coloring, pointed out that values could be cheaply recomputed versus spilled and refetched from memory. Based on this observation, they proposed a technique called rematerialization. This technique was later extended by Briggs et al. [9].

The work most closely related to our instruction replication technique is the work by Kuras et al. [27], where they describe a technique called value cloning for Long Instruction Word architectures with partitioned register banks. Their work targeted read-only values and induction variables.

Another approach used to address excess communications in clustered architectures is loop unrolling. There has been a significant amount of prior work addressing this topic, including techniques targeting clustered VLIW architectures [35]. Although unrolling can remove most of the communications and achieve high performance, it increases code size significantly. For DSPs, where VLIW architectures are frequently used [36], [19], [31], [17], [20], code size is a critical issue.

Cluster microarchitectures are also popular for dynamically scheduled processors. In this area, Aggarwal and Franklin [1] studied a technique to perform dynamic instruction replication.

Task duplication [26] has been used in the multiprocessors domain to alleviate the overhead introduced when tasks executing on different processors exchange data.

\section{Conclusions}

In this paper, we have presented AGAMOS, a scheme to modulo schedule loops for clustered microarchitectures. The proposed technique achieves improved workload balance and reduces the communications by using a multilevel graph partitioning strategy. In addition, in order to further reduce the communications, it performs instruction replication and schedules communications through memory.

AGAMOS is a two-step approach that overcomes the limitations of previous techniques by combining the benefits of the global view of the DDG obtained during the partition with the information available at scheduling time. In order to deal with the phase-ordering problem, pseudoschedules are used to guide cluster assignment and information from the partition is passed to the final scheduler. The partition can be modified at scheduling time.

The described technique is shown to outperform a state-ofthe-art scheduler for all programs and all configurations evaluated. Some of the speedups achieved are higher than 100 percent. Besides, 50 percent speedup is reached for many programs. Finally, we have also shown that by combining instruction replication and communications through memory, we can provide significant performance benefits.

\section{ACKNOWLEDGMENTS}

This work is supported by the Spanish Ministry of Education and Science and FEDER funds of the EU under contracts TIN 2004-03072, and TIN 2004-07739-C02-01, the Generalitat de Catalunya under grant 2005SGR00950, and Intel Corporation.

\section{REFERENCES}

[1] A. Aggarwal and M. Franklin, "Instruction Replication: Reducing Delays Due to Inter-PE Communication Latency," Proc. Int'l Conf. Parallel Architectures and Compiler Techniques (PACT '03), Sept. 2003.

[2] A. Aletà, J.M. Codina, J. Sánchez, and A. González, "GraphPartitioning Based Instruction Scheduling for Clustered Processors," Proc. 34th Int'l Symp. Microarchitecture, Dec. 2001.

[3] A. Aletà, J.M. Codina, J. Sánchez, A. González, and D. Kaeli, "Exploiting Pseudo-Schedules to Guide Data Dependence Graph Partitioning," Proc. Int'l Conf. Parallel Architectures and Compiler Techniques (PACT '02), Sept. 2002.

[4] A. Aletà, J.M. Codina, A. González, and D. Kaeli, "Instruction Replication for Clustered Microarchitectures," Proc. 36th Int'l Symp. Microarchitecture, 2003.

[5] A. Aletà, J.M. Codina, A. González, and D. Kaeli, "Removing Communications in Clustered Microarchitectures through Instruction Replication," ACM Trans. Architecture and Code Optimization (TACO), vol. 1, no. 2, pp. 127-151, June 2004.

[6] A. Aletà, J.M. Codina, A. González, and D. Kaeli, "Heterogeneous Clustered VLIW Microarchitectures," Proc. Fifth Int'l Symp. Code Generation and Optimization, pp. 354-366, 2007.

[7] J. Allen, K. Kennedy, and J. Warren, "Conversion of Control Dependence to Data Dependence," Proc. 10th Ann. Symp. Principles of Programming Languages, Jan. 1983.

[8] E. Ayguadé, C. Barrado, A. González, J. Labarta, D. López, S. Moreno, D. Papua, F. Reig, Q. Riera, and M. Valero, "Ictineo: A Tool for Research on ILP," Proc. Conf. Supercomputing, 1996.

[9] P. Briggs, K.D. Cooper, and L. Torczon, "Rematerialization," Proc. Special Interest Group on Programming Languages (SIGPLAN '92) Conf. Programming Language Design and Implementation, June 1992.

[10] A. Capitanio, N. Dutt, and A. Nicolau, "Partition Register Files for VLIW's: A Preliminary Analysis of Tradeoffs" Proc. 25th Int'l Symp. Microarchitecture (MICRO-25), 1992.

[11] G.J. Chaitin, M.A. Auslander, A.K. Chandra, J. Cocke, M.E. Hopkins, and P.W. Markstein, "Register Allocation via Coloring," Computer Languages, pp. 47-57, Jan. 1981.

[12] A. Charlesworth, "An Approach to Scientific Array Processing: The Architectural Design of the AP120B/FPS-164 Family," Computer, vol. 14, no. 9, pp. 18-27, Sept. 1981.

[13] M.L. Chu and S.A. Mahlke, "Compiler-Directed Data Partitioning for Multicluster Processors," Proc. Int'l Symp. Code Generation and Optimization (CGO), 2006.

[14] J.M. Codina, J. Sánchez, and A. González, “A Unified Modulo Scheduling and Register Allocation Technique for Clustered Processors," Proc. Int'l Conf. Parallel Architectures and Compilation Techniques, 2001. 
[15] J.M. Codina, J. Llosa, and A. González, “A Compartive Study of Modulo Scheduling Techniques," Proc. Int'l Conf. Supercomputing (ICS '02), June 2002.

[16] A.E. Eichenberger, E.S. Davidson, and S.G. Abraham, "Optimum Module Schedules for Minimum Register Requirements," Proc. Conf. Supercomputing, 1995.

[17] P. Faraboschi, G. Brown, J. Fisher, G. Desoli, and F. Homewood, "Lx: A Technology Platform for Customizable VLIW Embedded Processing," Proc. 27th Int'l Symp. Computer Architecture, June 2000.

[18] M.M. Fernandes, J. Llosa, and N. Topham, "Distributed Modulo Scheduling," Proc. Int'l Symp. High-Performance Computer Architecture, pp. 130-134, Jan. 1999.

[19] J. Fridman and Z. Greenfield, "The TigerSharc DSP Architecture," IEEE Micro, vol. 20, no. 1, pp. 66-76, Jan./Feb. 2000.

[20] P.N. Glaskowsky, "MAP1000 Unfolds at Equator," Microprocessor Report, vol. 12, no. 16, Dec. 1998.

[21] J. Hiser, S. Carr, P.H. Sweany, and S.J. Beaty, "Register Assignment for Software Pipelining with Partitioned Register Banks," Proc. 14th Int'l Parallel and Distributed Processing Symp., 2000.

[22] R. Ho, K. Mai, and M. Horowitz, "The Future of Wires," Proc. IEEE, pp. 490-504, Apr. 2001.

[23] S. Jain, "Circular Scheduling: A New Technique to Perform Software Pipelining," Proc. Int'l Conf. Programming Languages, Design and Implementation, 1991.

[24] G. Karpis and V. Kumar, "Analysis of Multilevel Graph Partitioning," Proc. Seventh Supercomputing Conf., 1995.

[25] B. Kernighan and S. Lin, "An Effective Heuristic Procedure for Partitioning Graphs," Bell Systems Technical J., 1970.

[26] B. Kruatrachue and T.G. Lewis, "Grain Size Determination for Parallel Processing," IEEE Software, vol. 5, no. 1, pp. 23-32, Jan. 1988.

[27] D. Kuras, S. Carr, and P. Sweany, "Value Cloning for Architectures with Partitioned Register Banks," Proc. Workshop Compiler and Architecture Support for Embedded Systems, pp. 1-5, Dec. 1998.

[28] J. Llosa, E. Ayguadé, A. González, and M. Valero, "Swing Modulo Scheduling," Proc. Int'l Conf. Parallel Architectures and Compilation Techniques (PACT '96), Oct. 1996.

[29] E. Nystrom and A.E. Eichenberger, "Effective Cluster Assignement for Modulo Scheduling," Proc. 31st Int'l Symp. Microarchitecture, pp. 103-114, 1998.

[30] E. Ozer, S. Banerjia, and T.M. Conte, "Unified Assign and Schedule: A New Approach to Scheduling for Clustered Register File Microarchitectures," Proc. 31st Int'l Symp. Microarchitecture (MICRO-31), 1998.

[31] G.G. Pechanek and S. Vassiliadis, "The ManArray Embedded Processor Architecture," Proc. 26th. Euromicro Conf.: "Informatics: Inventing the Future, "Sept. 2000.

[32] B.R. Rau and C. Glaeser, "Some Scheduling Techniques and an Easily Schedulable Horizontal Architecture for High Performance Schientific Computing," Proc. 14th Ann. Microprogramming Workshop, pp. 183-197, Oct. 1981.

[33] B.R. Rau, Iterative Modulo Scheduling. Hewlett-Packard Company, 1995.

[34] J. Sánchez and A. González, "Cache Sensitive Modulo Scheduling," Proc. 30th Int'l Symp. Microarchitecture, 1997.

[35] J. Sánchez and A. González, “The Effectiveness of Loop Unrolling for Modulo Scheduling in Clustered VLIW Architectures," Proc. 29th Int'l Conf. Parallel Processing, Aug. 2000.

[36] Texas Instruments, Inc., "TMS320C62x/67x CPU and Instruction Set Reference Guide," 1998.

[37] J. Zalamea, J. Llosa, E. Ayguadé, and M. Valero, "Modulo Scheduling with Integrated Register Spilling for Clustered VLIW Architectures," Proc. 34th Int'l Symp. Microarchitecture, Dec. 2001.

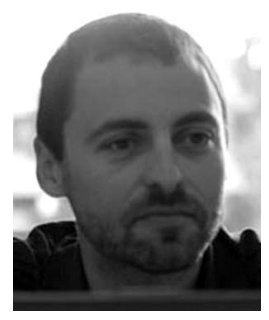

Alex Aletà received the Master's degree in mathematics from the Universitat Politècnica de Catalunya (UPC), where he is currently working toward the $\mathrm{PhD}$ degree in computer architecture. Then, he joined ARCO research group in the same university. He will graduate in 2009. His research has been focused on code generation and optimization for clustered VLIW microarchitectures.

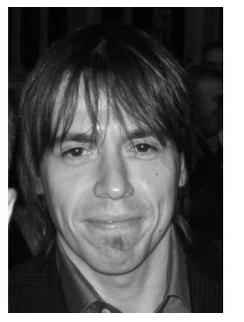

Josep M. Codina received the MS and PhD degrees in computer science from the Universitat Politècnica de Catalunya. He joined Intel in 2004, and he is a senior research scientist at Intel Barcelona Research Center. His research interests include computer architecture, compilers, code generation, and dynamic optimization for future microprocessors.

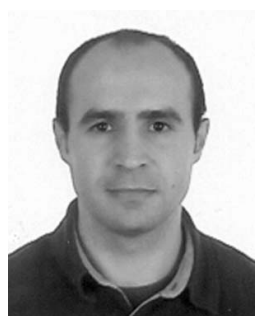

Jesús Sánchez received the $\mathrm{MS}$ and $\mathrm{PhD}$ degrees in computer engineering from the Universitat Politècnica de Catalunya (UPC), Barcelona, in 1995 and 2001, respectively. He joined the Department of Computer Arquitecture, UPC, in 1995, as a research assistant and was an assistant professor from 1998 to 2002. Since March 2002, he has been with the IntelUPC Barcelona Research Center, which he joined as a senior research scientist. His interests include processor microarchitecture and compilation techniques, in particular memory hierarchy, instruction-level parallelism, clustered architectures, instruction scheduling, and speculative multithreading. He has more than 30 publications on these topics. He is currently working on speculative multithreading techniques and FPGAbased prototypes. He is a member of the IEEE.

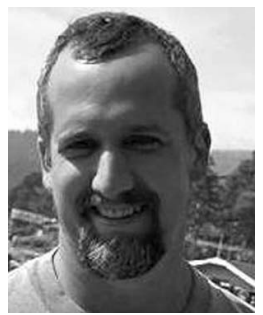

Antonio González received the MS and PhD degrees from the Universitat Politècnica de Catalunya (UPC), Barcelona, Spain. He is the founding director of the Intel Barcelona Research Center, started in 2002. His research focuses on new microarchitecture paradigms and code generation techniques for future microprocessors. Prior to this, he joined the faculty of the Computer Architecture Department of UPC in 1986, and became a full professor in 2002. He has published more than 300 papers, has given more than 80 invited talks, has filed more than 40 patents, and has advised $15 \mathrm{PhD}$ thesis in the areas of computer architecture and compilers. He has served as an associate editor of the IEEE Transactions on Computers, the IEEE Transactions on Parallel and Distributed Systems, ACM Transactions on Architecture and Code Optimization, and Journal of Embedded Computing. He has served on more than 100 program committees for international symposia in the field of computer architecture, including ISCA, MICRO, ASPLOS, HPCA, PACT, ICS, ISPASS, CASES, and IPDPS. He has been the program chair for ICS 2003, ISPASS 2003, MICRO 2004, and HPCA 2008, and the general chair for MICRO 2008, among other symposia.

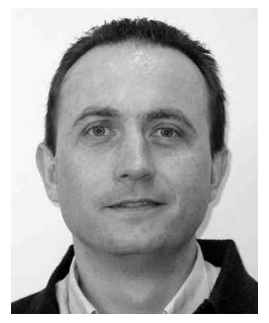

David Kaeli received the BS and $\mathrm{PhD}$ degrees in electrical engineering from Rutgers University, and the MS degree in computer engineering from Syracuse University. He is presently a full processor on the ECE Faculty at Northeastern University, Boston, MA, where he directs the Northeastern University Computer Architecture Research Laboratory (NUCAR). Prior to joining Northeastern in 1993, he spent 12 years at IBM, the last 7 at T.J. Watson Research Center, Yorktown Heights, NY. He has published more than 180 critically reviewed publications, five books, and eight patents. He regularly provides tutorials on the subject of profiling and instrumentation, and has organized workshops on GPGPUs, security, and binary translation. His research spans a range of areas including VLSI design to back-end compilers and database systems. He is an associate editor of the IEEE Computer Architecture Letters and the Journal of Instruction Level Parallelism, and the chair of the IEEE Technical Committee on Microprocessors and Microprogramming. He is also a member of CRA's Computing Consortium Council.

$\triangleright$ For more information on this or any other computing topic, please visit our Digital Library at www.computer.org/publications/dlib. 